\title{
Zukunftsfähige Formate für digitale Lernangebote - innovative didaktische Ansätze am Beispiel einer Lernplattform für Künstliche Intelligenz
}

\section{Dana-Kristin Mah und Julia Hense}

\section{Zusammenfassung}

Lernangebote müssen hochwertig und didaktisch innovativ sein, um Lernenden mit ihrem individuellen Bedarf gerecht $\mathrm{zu}$ werden. Für digitale Lernangebote gilt dies in besonderem Maße, da zum Beispiel die Möglichkeit des direkten Austauschs wie in Präsenzformaten nicht gegeben ist. Gleichzeitig ermöglichen digitale Lernangebote ein hochgradig individualisiertes und personalisiertes Lernangebot. Künstliche Intelligenz (KI) gewinnt im Alltag und auch als Lerninhalt an Bedeutung. KI findet zunehmend in alle Lebensbereiche Eingang. Entsprechend wichtig ist die Beschäftigung mit der Thematik ebenso wie die Vermittlung von KI-Kompetenzen, um verstehen zu können, wie KI funktioniert. Im Fokus des Beitrags steht deshalb die Frage, welchen didaktisch-methodischen Anforderungen eine digitale LehrLern-Plattform zum Thema KI gerecht werden muss. Dies wird anhand einer Analyse digitaler Lern-/Lehrformate auf etablierten digitalen Lernplattformen diskutiert. Im Fokus steht dabei die Frage, welche didaktischen Lern-/Lehrformate und Lernsettings geeignet sein könnten, um eine Stärkung von KI-Kompetenzen durch vorrangig digitale Lernangebote zu erreichen. In

D.-K. Mah (凶)

Stifterverband, KI-Campus, Berlin, Deutschland

E-Mail: dana-kristin.mah@stifterverband.de

J. Hense

mmb Institut, Essen, Deutschland

E-Mail: hense@mmb-institut.de 
der Diskussion werden Implikationen der Ergebnisse unter Berücksichtigung der Gestaltung und des Aufbaus der digitalen Lernplattform „KI-Campus - Die Lernplattform für Künstliche Intelligenz" skizziert.

\section{Schlüsselwörter}

Künstliche Intelligenz $\bullet$ KI-Kompetenzen $•$ Digitales Lernen $\bullet$ Digitale

Didaktik $\bullet$ Digitale Lernformate $\bullet$ Innovative Lernformate

\section{Einleitung: Sag, wie hältst du's mit dem digitalen Lernen?}

In den letzten Jahren hat sich mit Blick auf digitales Lernen an deutschen Hochschulen einiges bewegt: Die Förderlinie Digitale Hochschulbildung wurde vom Bundesministerium für Bildung und Forschung (BMBF) initiiert, die Digital Learning Map (Hochschulforum Digitalisierung 2020) listet in einer Datenbank über 120 Praxisbeispiele innovationsfreudiger Vorhaben und Good-PracticeBeispiele digitaler Hochschullehre in Deutschland auf. Der DigitalPakt für Schulen (BMBF 2019) wurde verabschiedet und Lernplattformen, die sowohl breit ausgerichtete Kurse zur eigenen Weiterbildung als auch Angebote auf Hochschulniveau zur Verfügung stellen, erfreuen sich großer Beliebtheit (Seyda et al. 2018). Zudem erfährt die deutsche Hochschullehre durch die Bund-Länder-Vereinbarung „Innovation in der Hochschullehre“ besondere Aufmerksamkeit. Trotz dieser Initiativen besteht weiterhin Handlungsbedarf.

Dieser Handlungsbedarf zeigt sich eindringlich zu Beginn der Coronakrise im Frühjahr 2020, die eine rasante Umstellung der Hochschulen von Präsenzauf Onlineveranstaltungen erfordert. Technische und infrastrukturelle Defizite vieler Hochschulen treffen auf vielfältige technische und didaktische Herausforderungen bei der Konzeption und Durchführung von Onlineveranstaltungen. Das Erfordernis, rasant auf digitale Lernformate für die Hochschulbildung umzustellen, kann auch als Katalysator und Chance betrachtet werden. Die Nachfrage nach virtuellen Austauschmöglichkeiten und digitalen Lernangeboten steigt massiv, insbesondere vor dem Hintergrund der aktuellen Entwicklungen (Burchard 2020). Die komplexen Herausforderungen der digitalen Hochschulbildung in Deutschland zur Coronakrise werden nun auch für eine breitere Öffentlichkeit offenkundig, beispielsweise in der Diskussion eines Nichtsemesters für das Sommersemester 2020 (https://www.nichtsemester.de/cbxpetition/offener-brief/). Die Länder haben sich auf ein gemeinsames Vorgehen zum Sommersemester 2020 
verständigt; demnach werden unter anderem die Semesterzeiten für das Sommersemester 2020 nicht verschoben (Kultusministerkonferenz 2020), ,Vorlesungen und Seminare finden nurmehr digital statt“", erklärt zudem der Präsident der Hochschulrektorenkonferenz (2020). Eine zusätzliche Sonderbefragung zur jährlichen Hochschul-Barometer-Befragung des Stifterverbands im April 2020 hat ergeben, dass sich insgesamt $90 \%$ der 168 teilnehmenden Hochschulen als ,gut gerüstet für den Semesterstart" mit Blick auf ihr digitales Lehrangebot einschätzen; $76 \%$ der Lehre könnten angeboten werden (Stifterverband für die Deutsche Wissenschaft e. V. 2020). Der ,Ausbau mediendidaktischer Qualifizierung von Lehrenden und E-Learning-Support“ wird nach „Ausbau IT-Infrastruktur und -Ausstattung“ als wichtigste Sofortmaßnahme bewertet (Stifterverband für die Deutsche Wissenschaft e. V. 2020).

Folglich ist zu erwarten, dass digitalisierte Lernangebote in nächster Zeit noch eine deutliche Stärkung erfahren werden. Umso wichtiger ist es, einen Überblick über die aktuellen innovativen digitalen Lernformate $\mathrm{zu}$ geben und auch neuere Entwicklungen einzubeziehen, wie zum Beispiel die Möglichkeiten von Künstlicher Intelligenz (KI). Auf der Basis müssen Leitlinien entwickelt und ausgegeben werden, um zu zeigen, was gute digitale Lernangebote aus didaktischer Sicht ausmacht. Bislang bedauerte die Expertenkommission Forschung und Innovation (EFI) in ihrem Gutachten von 2019 ,die Zurückhaltung deutscher Hochschulen bei der systematischen Entwicklung und Bereitstellung innovativer digitaler Bildungs- und Weiterbildungsangebote" (EFI 2019, S. 99). Beispielsweise würden deutsche Hochschulen im internationalen Vergleich bislang kaum offen zugängliche Lernangebote auf digitalen Lernplattformen offerieren - gemeint sind damit Webportale wie Coursera oder Iversity, die (self-paced) Onlinekurse anbieten (EFI 2019). Das ist angesichts des noch immer nicht ausreichenden Ausbaus der notwendigen digitalen Infrastruktur in Deutschland und der langen Entwicklungszeiten im Bildungssektor wenig verwunderlich. Jedoch ist gerade die digitale Welt eine schnelllebige, die sich rasant entwickelt und auch im Bildungssektor zunehmend an Bedeutung gewinnt. Deutschland ist, was die Nutzung von digitalen Medien für Lernprozesse betrifft, im internationalen Vergleich eher Schlusslicht. Staaten wie Estland, Finnland oder auch Singapur sind uns hier weit voraus sowohl was die Nutzung digitaler Lernmedien betrifft als auch die Offenheit, neue Formate auszuprobieren und neue Technologien zu nutzen (Forum Bildung Digitalisierung 2019).

In jüngster Zeit wird dies auch in der Debatte um Künstliche Intelligenz (KI) deutlich. Die Möglichkeiten, die KI bietet, sind vielfältig und werden in vielen Ländern auch zunehmend genutzt. Umso wichtiger ist es, die Möglichkeiten 
und Chancen, aber auch Grenzen und Herausforderungen rund um KI wahrzunehmen, zu reflektieren und bewusst zu gestalten. Das geschieht in Deutschland zunehmend, jedoch insbesondere im Bildungssektor noch nicht ausreichend. Die Relevanz und Aktualität des Themas KI in der Hochschulbildung zeigt sich dennoch an zunehmenden Aktivitäten und Förderprogrammen in Deutschland, zum Beispiel an der im Februar 2020 veröffentlichten ,Richtlinie zur Förderung von Zuwendungen für die Forschung zur digitalen Hochschulbildung - Innovationen in der Hochschulbildung durch Künstliche Intelligenz und Big Data" des BMBF (2020) oder dem Whitepaper „Künstliche Intelligenz in der Hochschulbildung“ (de Witt et al. 2020). Neben der Förderung notwendiger vermehrter Forschungsaktivitäten zu KI in der Hochschulbildung reagiert das Bundesministerium für Bildung und Forschung auf die Nachfrage nach praktischen Bildungsangeboten zum Thema KI mit der Förderung des Projekts „KI-Campus - Die Lernplattform für Künstliche Intelligenz" (https://ki-campus.org/).

Im Fokus dieses Beitrags stehen die Fragen, welche digitalen Lern-/ Lehrformate aktuell auf etablierten digitalen Lernplattformen angeboten werden und welche didaktischen Lern-/Lehrformate und Lernsettings geeignet sein könnten, um eine Stärkung von KI-Kompetenzen durch vorrangig digitale Lernangebote zu erreichen. Zunächst wird dafür die Bandbreite digitaler Lernplattformen in den Blick genommen. Betrachtet werden Gemeinsamkeiten sowie Besonderheiten didaktischer Ansätze ausgewählter digitaler Lernplattformen, um einen Überblick über aktuelle Entwicklungen im Bereich digitaler Didaktik zu erheben. In der Diskussion werden Implikationen der Ergebnisse unter Berücksichtigung der Gestaltung und des Aufbaus der digitalen Lernplattform KI-Campus skizziert.

\section{Digitale Lernangebote}

\subsection{Verschiedene didaktische Ansätze auf digitalen Lernplattformen}

Digitale Lernplattformen bieten Lernenden die Möglichkeit, flexibel zeit- und ortsunabhängig zu lernen - vorausgesetzt, das Angebot kann mit den verfügbaren technischen Mitteln aufgerufen werden. In Deutschland besteht hier, wie im Kap. 1 beschrieben, vielfach noch Nachholbedarf. Die Coronakrise hat gezeigt, wie schnell der reibungslose Zugriff auf digitale Lernmaterialien und die Verfügbarkeit didaktisch gut aufbereiteter digitaler Lernmedien sehr wichtig werden kann. Neben relevanten und qualitativ hochwertigen Lerninhalten sind didaktisch innovative und zukunftsfähige Lehr- und Lernformate von zentraler Bedeutung, 
um Lernenden mit ihrem individuellen Bedarf, ihren Präferenzen und Lernerfahrungen gerecht $\mathrm{zu}$ werden. $\mathrm{Zu}$ den größten und bekanntesten Lernplattformen zählen international Coursera, edX und Udacity (Shah 2019a). Coursera ist mit 45 Mio. registrierten Lernenden und 3800 Kursangeboten im Jahr 2019 der größte Anbieter von Onlinelernangeboten (Shah 2019b). Beispiele für deutsche Lernplattformen sind OpenHPI, Hamburg Open Online University (HOOU) und oncampus (Schmid et al. 2018).

Vielen digitalen Lernplattformen ist gemeinsam, dass sie Onlinekurse anbieten, die für eine große Zahl von Lernenden ausgelegt sind. Ein zentraler Bestandteil der Kurse auf solchen digitalen Lernplattformen ist das Lernvideo: Zu einem Kurs gehört in den meisten Fällen eine Reihe von Lernvideos, die von den Dozierenden eigens dafür erstellt und zusammengestellt wurden, um die Inhalte zu vermitteln. Je nach Ausrichtung des Kurses und der Plattform sind weitere Funktionen gegeben, wie zum Beispiel die Möglichkeit, zusätzliche Aufgaben zum Kurs im Selbststudium zu erarbeiten, sich in einem Forum mit anderen Kursteilnehmenden auszutauschen oder auch in Live-Webinaren direkt mit anderen Kursteilnehmenden und den Dozierenden via Videokonferenz in Kontakt zu treten. Wer die Anfänge der MOOCs (Massive Open Online Courses) verfolgt hat, sieht hier die Weiterentwicklung der Idee. Anstelle einer einzelnen abgefilmten Vorlesung gibt es eigens erstellte Videos, die speziell für das digitale Lernen gedreht wurden und von weiteren Maßnahmen flankiert werden, um den Lernprozess zu begleiten und Kursabbrüche gering zu halten (Hüther et al. 2020). Moderne Lernplattformen haben darauf reagiert, dass Lernen auch im digitalen Zeitalter ein sozialer Prozess ist, der für jeden Menschen unterschiedlich verläuft. Entsprechend müssen digitale Lernangebote mehr noch als analoge Lernangebote gut strukturiert, ansprechend gestaltet und für viele Lerntypen geeignet sein (Goertz 2014). Idealerweise sind digitale Lernplattformen klar strukturiert und intuitiv $\mathrm{zu}$ bedienen (ebenda). Sie bieten gut und ansprechend aufbereitete Inhalte, die für diverse Lernpräferenzen geeignet sind und individuelle Lernwege erlauben. Zusätzlich stehen Support, Betreuung, die Möglichkeit zum Austausch, Feedback und eine regelmäßige motivierende Erfolgskontrolle zur Verfügung. Das Lernvideo ist in der Regel ein zentraler Bestandteil des Lernangebots. Aufbau und Gestaltung der Videos variieren und werden durch Quiz, Download-Material, Tutoring-Angebote oder auch Prüfungs- und Zertifizierungsmöglichkeiten ergänzt. Je nach Zielgruppe und thematischer Ausrichtung der Lernplattform dominieren MOOCs wie bei Coursera und Udacity oder exklusivere Kursformate mit einer engen tutoriellen Begleitung der Lernenden, wie sie bei GoogleSquared zu finden sind (https://www.weares quared.de/). Derzeit zeichnet sich nach Expert*innenmeinung ein Shift hin zur Learning Experience Platform (LXP) ab (zum Beispiel Bersin 2018), bei der 
eine motivierende, spannende personalisierte Lernerfahrung im Vordergrund steht. Ein Beispiel dafür ist die spanischsprachige Plattform Domestika, auf der alle Kursteilnehmenden auf einem Marktplatz eigene Projekte präsentieren können, sich vernetzen sowie Arbeitsproben für potenzielle Arbeitgeber vorstellen (https:// www.domestika.org/). Die Lernplattform LabXChange setzt auf Microcontent, den engen Austausch der Lerncommunity untereinander und die gemeinsame Erstellung von Inhalten oder auch die Umsetzung eigener Projekte im Rahmen der Lernprozesse, die wiederum anderen Lernenden zur Verfügung gestellt werden können (https://www.labxchange.org/). Das auf anderen Plattformen häufig noch vorzufindende klare Kurssystem, das sich an einem von Lehrenden vorstrukturierten Lernprozess orientiert, wird in beiden Beispielen zunehmend zugunsten eines lernendenzentrierten selbstgesteuerten Lernprozesses aufgelöst, der deutlich die Eigenorganisation der Lernenden akzentuiert und durch ansprechende, frei zu bearbeitende Inhalte fast schon spielerisch aufgebaut ist.

\subsection{Digitale Lernangebote zum Thema Künstliche Intelligenz}

In der „Strategie Künstliche Intelligenz“ der Bundesregierung wird betont, dass keine ,einzige allgemeingültige bzw. von allen Akteuren konsistent genutzte Definition von KI gibt" (Die Bundesregierung 2018, S. 4). Gleichwohl wird die Unterscheidung zwischen sogenannter starker KI und schwacher KI betont. Die starke KI bezieht sich auf KI-Systeme mit den gleichen intellektuellen Fähigkeiten wie Menschen oder sogar übertreffenden intellektuellen Fähigkeiten. Demgegenüber steht die schwache KI, die sich - basierend auf mathematischen und informatischen Methoden, die zur Selbstoptimierung fähig sind - auf die Lösung konkreter Anwendungsprobleme bezieht. Die Bundesregierung orientiert sich bei ihrer KI-Strategie an der „schwachen KI“ (Die Bundesregierung 2018, S. 5). In Deutschland sowie international zeigt sich ein großer Bedarf an Fachkräften im Bereich KI. Im Jahr 2019 setzten rund 17.500 Unternehmen in Deutschland, das heißt 5,8 \% der Unternehmen im Berichtskreis der Innovationserhebung, KI in Produktion, Dienstleistungen oder internen Prozessen ein. Dabei suchen $30 \%$ der KI-einsetzenden Unternehmen zusätzliche Beschäftigte für KI - von den insgesamt 22.500 offenen KI-Stellen sind $43 \%$ unbesetzt (BMWi 2020). Um die wissenschaftliche Nachwuchsförderung im Bereich KI zu befördern, strebt die Bundesregierung die Schaffung von „mindestens 100 neuen Professuren“ (Die Bundesregierung 2018, S. 15) bis zum Jahr 2025 an - laut „Zwischenbericht: Ein Jahr KI-Strategie“" wurden 30 KI-Professuren für Deutschland weltweit ausgeschrieben (Die Bundesregierung 2019). Die EFI-Expertenkommission bewertet 
das Ziel der 100 neuen KI-Professuren vor dem Hintergrund des weltweiten Wettbewerbs um KI-Fachkräfte jedoch als sehr ambitioniert (EFI 2019). An deutschen Hochschulen existierten im Jahr 2019 mindestens 192 Professuren mit einem KISchwerpunkt und mindestens 75 Studienangebote mit KI-Schwerpunkt (Mah und Büching 2019). Digitale Lernangebote zum Thema KI werden derzeit vorwiegend auf internationalen Lernplattformen angeboten, wie Coursera und edX (Mah et al. 2020). In Europa ist vor allem der kostenfreie finnische Onlinekurs Elements of AI populär. Der Onlinekurs der Universität Helsinki setzt vollständig auf Text und eine ansprechende grafische Gestaltung der Lerninhalte. Die Inhalte sind in einzelne Kapitel unterteilt. Am Ende jedes Kapitels wird der Wissenserwerb durch ein Quiz überprüft. Seit Ende 2019 ist der Onlinekurs auf Deutsch verfügbar; demnächst soll er in allen EU-Sprachen erhältlich sein (https://www.elementso fai.com/).

\subsection{KI-Campus - Die Lernplattform für Künstliche Intelligenz}

Um einen mündigen und kompetenten Umgang mit Künstlicher Intelligenz (KI) zu stärken und mittelfristig auch einem akuten Fachkräftemangel zu begegnen, fördert das Bundesministerium für Bildung und Forschung seit Oktober 2019 das Pilotvorhaben „KI-Campus - Die Lernplattform für Künstliche Intelligenz“ (https://www.ki-campus.org/). Im Zentrum steht der prototypische Aufbau einer auf das Thema Künstliche Intelligenz (KI) spezialisierten digitalen Lernplattform (Rampelt et al. 2019). Die Lernangebote zu KI umfassen Grundlagen, bereichsspezifische KI-Themen und KI-Anwendungen sowie interdisziplinäre KIFragestellungen. Das didaktische Konzept für die Entwicklung des KI-Campus sieht zukunftsfähige, innovative und soziale Lernformate vor, die die Lernenden und ihre Lernprozesse in den Mittelpunkt der Lernangebote stellen (Shift from Teaching to Learning).

\section{Forschungsfragen und methodisches Vorgehen}

Folgende Forschungsfragen werden in diesem Beitrag mit Blick auf zukunftsfähige Formate für digitale Lernangebote zur Stärkung von KI-Kompetenzen adressiert: 
1. Was sind bekannte/etablierte internationale und nationale themenoffene digitale Lernplattformen für die Zielgruppen Studierende und lebenslang Lernende?

2. Welche Lernplattformen bieten welche zukunftsfähigen Lern-/Lehrformate für digitale Lernangebote? Was sind Gemeinsamkeiten und Besonderheiten?

Diese Fragen wurden ausgewählt, um zunächst einen fundierten Überblick über bestehende digitale Lernplattformen und Lernangebote zu erhalten. Dazu wurden Ist-Analysen (Desk Research) des State of the Art virtueller Lernorte mit Blick auf didaktische Aspekte diverser digitaler Lernplattformen vorgenommen.

Basierend auf den Analyseergebnissen wird in der Diskussion folgende weitere Frage adressiert:

3. Welche didaktischen Lern- und Lehrformate und Lernsettings könnten geeignet sein, um eine Stärkung von KI-Kompetenzen durch vorrangig digitale Lernangebote zu erreichen?

\section{$4 \quad$ Ergebnisse}

Für die Analyse des State of the Art virtueller Lernorte wurden verschiedene digitale Lernplattformen und digitale Lernangebote systematisch gesichtet und unter didaktischen Gesichtspunkten analysiert. Ein Kriterium für die Auswahl der Angebote war einerseits die Einordnung als digitale Lernplattform in Anlehnung an Schulmeister (2005) oder die Einordnung als digitales Kursangebot zum eigenständigen Onlinelernen im Sinne eines (self-paced) MOOCs. Dabei erfolgte die Auswahl breit, ohne einen besonderen thematisch-inhaltlichen Fokus des Angebots vorauszusetzen, ebenso wie gezielt im Hinblick auf Ansätze von Angeboten zum Lernen mit und über KI. Die Auswahl der betrachteten digitalen Lernplattformen (Forschungsfrage 1) fiel so auf Coursera, MasterClass, oncampus, Udacity, xuetangx, EdX, OpenHPI, GoogleSquared, FutureLearn, Iversity, LabXchange, Domestika, Linkedin Learning, HOOU und Elements of AI.

Für die systematische Analyse dieser Lernplattformen mit Blick auf didaktische Ansätze und zukunftsfähige Formate der digitalen Lernangebote (Forschungsfrage 2) wurden 1) Gemeinsamkeiten und 2) Besonderheiten untersucht. Da die Analyse als Desk Research durchgeführt wurde, beziehen sich die Ergebnisse auf die auf den Plattformen selbst recherchierbaren Informationen. Zur Einordnung der Informationen wurde ein Kriterienraster mit insgesamt 15 didaktischen Kriterien entwickelt und für die Analyse zugrunde gelegt. Kriterien, die hier 
eingeflossen sind, waren zum Beispiel verfügbare Informationen zur Zielgruppe eines Lernangebots, zu Inhalten, Lernformaten, Prüfungsformaten, aber auch zu Dozierenden, Kursstrukturen und zusätzlichen Angeboten wie zum Beispiel Coaching, Tutoring oder der Möglichkeit, sich in einer Community auszutauschen. Nachfolgend werden besonders interessante Ergebnisse der Analyse präsentiert.

(1) Insgesamt zeigt die Analyse, dass sich viele Angebote in ihren didaktischen Ansätzen und im Aufbau ähneln. Gemeinsamkeiten der näher betrachteten Plattformen liegen insbesondere in der Bereitstellung von Lernvideos, die in Art und Ausgestaltung variieren, Download-Material für das Selbststudium, Lernstandskontrollen zum Beispiel in Form von Lernquiz und Austauschmöglichkeiten zwischen den Kursteilnehmenden und - wo angeboten - den Kursbetreuenden, zum Beispiel in Form eines Kursforums. Grundsätzlich sind die Kursbestandteile bei den untersuchten Lernplattformen modular aufgebaut und können häufig auch als Microcontent alleinstehen. Das erlaubt, zum Beispiel einzelne Lernvideos und die dazugehörigen Quizfragen auch alleinstehend zur Verfügung zu stellen oder in unterschiedlichen Kursen einzusetzen. So kann ein Inhalt in mehreren Kursen auch mit unterschiedlichen Zielgruppen und Ausrichtungen genutzt werden. Theoretisch erleichtert dieses Vorgehen auch den Aufbau individueller Lernpfade innerhalb eines Themas oder Kurses (zum Beispiel Udacity, Coursera, Iversity, EdX). Perspektivisch könnten bei einem solchen Ansatz sogar Kursteilnehmende eingebunden werden, indem sie eigene Playlists zu Themen erstellen, sich so ihre eigenen Lernpfade erstellen, die sie mit anderen teilen und ggf. sogar um weitere Inhalte ergänzen können, ganz im Sinne des „Lernens durch Lehren“. Auf der Plattform LabXchange können zum Beispiel schon einzelne Inhalte zu eigenen Listen zusammengestellt werden (https://www.labxchange.org/library). Verschiedene Inhalte werden eher häufiger zu Kursmodulen zusammengefasst, die wiederum in unterschiedlichen Kursen zum Einsatz kommen können und so zum Beispiel zu verschiedenen Zertifikatsprogrammen gebündelt werden. Bei den analysierten Lernplattformen sind die Kurse üblicherweise mit einem festen Startund Enddatum versehen und haben eine Laufzeit von circa drei bis zwölf Wochen. Je nach Kursangebot sind Angebote zum Tutoring - also die Möglichkeit jenseits der Kursinhalte in kleinen Gruppen mit einem Tutor konkrete Fragestellungen zum Kursthema durchzugehen - vorgesehen oder nicht. Manche Anbieter koppeln Tutoring-Angebote an Zertifikatskurse, die dann entsprechend teurer sind als Kursangebote ohne Tutoring und Zertifizierung. Andere Anbieter ermöglichen die Option, ein 1:1-Mentoring bei Bedarf individuell zuzubuchen. Mehrere Anbieter setzen auf Coaching- und Tutoring-Angebote, um Lernende individuell unterstützen zu können. Inzwischen gibt es sogar übergreifende Plattformen, die diesen 
Service anbieten, wenn eine entsprechende Begleitung nicht zum Kurs gehört. Hier können sich die Studierenden ihren eigenen Tutor oder ihre Tutorin aussuchen, die sie aufgrund des fachlichen Hintergrunds und einer Profilbeschreibung für passend halten (zum Beispiel https://www.wyzant.com/; https://www.skooli. com/). Nach Stand der Analyse lässt sich sagen: Je aufwendiger Inhalte produziert sind und je mehr Begleitangebote es in einem Kurs gibt (zum Beispiel Tutoring, Coaching, Prüfungsbegleitung), desto teurer ist der Kurs auch und desto eher steht das Ziel eines Zertifikatserwerbs dahinter.

(2) Neben diesen grundsätzlichen Tendenzen ließen sich bei einigen Angeboten für digitale Lernplattformen neue(re) didaktische Ansätze finden. So arbeitet Udacity zum Beispiel mit praktischen Projekten, die über die Laufzeit eines Kurses von den Kursteilnehmenden erarbeitet werden müssen. Das kann einzeln geschehen oder als Lerngruppe im Kurs, selbst initiiert oder auch unter Beteiligung von Unternehmen, die eine praktische Fragestellung haben. Die spanischsprachige Lernplattform Domestika, die sich auf den Designsektor spezialisiert hat, nutzt ebenfalls Kursprojekte und bietet den Kursteilnehmenden die Möglichkeit, ihre Portfolios offen auf der Webseite der Plattform auszustellen. Ein ähnlicher Ansatz kann auch für den KI-Sektor interessant sein, etwa wenn Kursteilnehmende in einem Kurs selbst Projekte programmiert haben. Angebote wie GoogleSquared setzen auf eine hochwertige individuelle Betreuung durch hochqualifizierte Lehrende und fokussieren sich dabei auf einzelne Kurse und Themen - das unterscheidet sie von Plattformangeboten wie Iversity, die breit Inhalte anbieten. Ein interessanter Sonderfall ist hier auch der finnische Kurs Elements of AI, ein digitales Lernangebot, das sich einzig mit dem Thema KI befasst, allerdings keinerlei Lernvideos oder Coachings im Programm hat, sondern allein auf Text und Quiz zur Lernstandskontrolle basiert. Damit hebt der Kurs sich didaktisch von den großen erkennbaren Strömungen im Hinblick auf digitale Lernangebote ab. Die Struktur ist klar und minimalistisch und der Erfolg des Kurses (University of Helsinki 2019) zeigt, dass auch dies ein gangbarer Weg ist. Als weiterer interessanter Ansatz für die didaktische Gestaltung digitaler Lernmaterialien sei auf die Nutzung eines Tag-Systems für Kurse und Kursbestandteile verwiesen, wie sie zum Beispiel bei oncampus zum Einsatz kommen. Hier werden Kurse mit einheitlichen Begriffen versehen, die mit der Suchfunktion verknüpft sind. Kursinteressierte können die Tags anklicken und so sehen, welche Inhalte auch unter diesem Tag gelistet sind. Das erleichtert die Suche und erlaubt auch eine flexible Vorgehensweise bei der Auswahl von Kursen. Denkt man dieses System weiter, so könnten zum Beispiel auch Kursteilnehmende beim Taggen von Kursen beteiligt werden. Auf diese Weise ließe sich unter Nutzung der Schwarmintelligenz mittelfristig ein stabiles Tag-System aufbauen, das 
- verknüpft mit Microcontent - zum Beispiel hilfreich wäre bei der Erstellung individueller Lernpfade.

\section{Diskussion, Implikationen und Ausblick}

Die Analyseergebnisse zeigen, dass es vielfältige und innovative didaktische Ansätze gibt, um digitale Lernangebote auf Onlinelernplattformen zu gestalten, für viele verschiedene Zielgruppen und Lernpräferenzen zugänglich zu machen und dabei auch neuere technische Anwendungen einzusetzen. Nachfolgend werden die Analyseergebnisse mit Blick auf ihre Implikationen für die Auswahl didaktischer Ansätze und Konzepte für den KI-Campus näher beleuchtet (Forschungsfrage 3).

Die große Verbreitung von Lernvideos, die mit weiteren Elementen wie zum Beispiel Quiz und Zusatzmaterialien angereichert werden, zeigt, dass dies ein tragfähiger Ansatz ist. Studien, die die Veränderungen im Bereich E-Learning in den Blick nehmen, legen dies ebenfalls nahe (mmb Institut 2020). Die Möglichkeiten, die zum Beispiel der Einsatz von H5P (https://h5p.org/) für die Gestaltung von Lernvideos bietet, sind hier noch nicht ausgeschöpft und werden auch noch nicht flächendeckend eingesetzt. H5P erlaubt beispielsweise eine flexible Handhabung von Lernvideos, das Einbetten von Quizfragen und die Gestaltung von individuellen Lernpfaden und stellt damit einen innovativen Ansatz für die Gestaltung von Lernvideos dar. Die Angebote für KI-Campus sollten sich grundsätzlich an der Bereitstellung von (1) Lernvideos orientieren. Weiterhin wird die aktive (Mit-)Gestaltung von Lernmaterialien durch die Nutzenden als ein wichtiges und noch ausbaufähiges Element betrachtet, das sich nicht nur aus grundsätzlichen wissenschaftlichen Erkenntnissen zur Motivation von Lernenden speist, sondern auch aus der eingangs erwähnten Beobachtung eines Shifts zur Learning Experience Platform, der die Mitwirkung der Lernenden als festen Bestandteil vorsieht (2). Microcontent als eigene Kategorie von Lernmaterial wird so verstärkt in den Blick genommen. Gleichermaßen legen die Analyseergebnisse nahe, dass (3) Betreuungs- und Tutoring-Angebote nicht unerheblich sind, insbesondere für Lernende, die Inhalte auf einem hohen fachlichen Niveau erarbeiten wollen, gegebenenfalls mit dem Anspruch, ein Zertifikat zu erwerben. Auch diesem Umstand sollte der KI-Campus Rechnung tragen. Entsprechend muss die didaktische Grundkonzeption ein Modell zur Umsetzung von begleitenden Austauschund Tutoring-Angeboten umfassen. Darüber hinaus hat die Analyse gezeigt, dass es sich lohnt, interaktive Lernvideos und Kursprojektarbeiten einzubeziehen. Dies 
kann helfen, individuelle Lernpfade zu ermöglichen (4). Für eine an sich klassische Lernplattform wie den KI-Campus, jedoch mit einer speziellen fachlichen Ausrichtung auf KI, kann das didaktisch durchaus interessant sein. Im Sinne der didaktischen Grundregel, Lernprozesse vom Leichten zum Schweren hin zu gestalten, können hier auch die Lernformate entsprechend gut angepasst werden. Denkbar sind zum Beispiel sehr anschauliche Kurse mit vielen interaktiven Lernvideos entlang eines vorbestimmten Lernpfads für Anfänger*innen über stärker individualisierbare Angebote zum Beispiel mit Microcontent bis hin zum Zertifikatsprogramm, bestehend aus Lernvideos und Texten auf akademischem Niveau mit starker tutorieller Begleitung und Kursprojekten auf praktischer Ebene. Für die Umsetzung erscheint es sinnvoll, zunächst mit einigen wenigen gut gestalteten Lernangeboten zu starten und diese dann sukzessive auszubauen. In didaktischer Hinsicht lässt sich dies zum Beispiel durch eine konsequent verfolgte Modularisierung von Inhalten erreichen. Des Weiteren sind Ansätze denkbar, die ein (5) Flipped-Classroom-Modell ermöglichen oder auch kollaboratives Arbeiten an Projekten bis hin zur stärker spielerischen Gestaltung von Lernsessions. Nicht zuletzt sind auch innovative Settings des Blended Learning denkbar, die virtuelle Lernangebote mit Workshops, Sprints oder BarCamps in der realen Welt verknüpfen.

Die dargestellten Analyseergebnisse können als erste Orientierung für die didaktische Gestaltung des KI-Campus dienen. Weitere Analysen und qualitative wie quantitative Forschung sind erforderlich, um valide Aussagen $\mathrm{zu}$ didaktischen Gelingensbedingungen zu erhalten. Im Fokus weiterer Forschung steht die Frage, welche (digitalen) Lernangebote mit Blick auf didaktische Ansätze, Lernformate und Lernsettings besonders geeignet sind, um Kompetenzen im Bereich KI zu stärken. Hierzu sind systematische Analysen weiterer (didaktischer) Kriterien erforderlich, wie etwa Zielgruppe der Lernangebote, avisierte Lernergebnisse und Kompetenzen, Einsatz von Learning Analytics, Qualifikation der Dozierenden sowie Prüfungsprozesse und Zertifizierungsmöglichkeiten. Zudem könnte mit Blick auf den KI-Campus untersucht werden, welche neuen Lernsettings und -elemente von welchen Lernenden mit welchem Hintergrund und welcher Zielstellung für die Teilnahme an Lernangeboten als hilfreich empfunden werden. Ein besonderer Fokus wird auf der Frage liegen, wie zum Beispiel der Einsatz von KI beim Erwerb von KI-Kompetenzen unterstützend wirken kann. Neben Learning Analytics (Büching et al. 2019; Ifenthaler und Schumacher 2016) sind unter anderem KI-basierte Chatbots, die Lernende in ihren Lernprozessen begleiten, denkbar (de Witt et al. 2020). Interdisziplinäre Zusammenarbeit in der Entwicklung und Anwendung von KI-Methoden zum Lernen und Lehren ist anzustreben (Zawacki-Richter et al. 2019). 


\section{Literatur}

\#nichtsemester. (2020). Das Sommersemester 2020 muss ein „Nichtsemester“ werden - Ein offener Brief aus Forschung und Lehre. https://www.nichtsemester.de/cbxpetition/offenerbrief/ Zugegriffen: 18. Nov. 2020.

Bersin, J. (2018). The Learning Experience Platform (LXP) market expands. https://jos hbersin.com/2018/09/the-learning-experience-platform-lxp-market-expands/. Zugegriffen: 18. Nov. 2020.

BMBF - Bundesministerium für Bildung und Forschung. (2019). Verwaltungsvereinbarung DigitalPakt Schule 2019 bis 2024. https://www.digitalpaktschule.de/de/was-ist-der-digita lpakt-schule-1701.html. Zugegriffen: 18. Nov. 2020.

BMBF - Bundesministerium für Bildung und Forschung. (2020). Richtlinie zur Förderung von Zuwendungen für die Forschung zur digitalen Hochschulbildung - Innovationen in der Hochschulbildung durch Künstliche Intelligenz und Big Data. https://www.bmbf.de/ foerderungen/bekanntmachung-2873.html. Zugegriffen: 18. Nov. 2020.

BMWi - Bundesministerium für Wirtschaft und Energie. (2020). Einsatz von Künstlicher Intelligenz, in der Wirtschaft. Stand der KI-Nutzung im Jahr 2019. Berlin: Bundesministerium für Wirtschaft und Energie.

Büching, C., Mah, D.-K., Otto, S., Paulicke, P., \& Hartman, E. A. (2019). Learning Analytics an Hochschulen. In V. Wittpahl (Hrsg.), Künstliche Intelligenz (S. 142-160). Berlin: Springer.

Burchard, A. (1. April 2020). Umstellung auf E-Learning in der Corona-Krise: Mutige Expeditionen ins Neuland. Der Tagesspiegel. https://www.tagesspiegel.de/wissen/ums tellung-auf-e-learning-in-der-corona-krise-mutige-expeditionen-ins-neuland/25702776. html. Zugegriffen: 18. Nov. 2020.

Die Bundesregierung. (2018). Strategie Künstliche Intelligenz der Bundesregierung. https:// www.bmbf.de/files/Nationale_KI-Strategie.pdf. Zugegriffen: 18. Nov. 2020.

Die Bundesregierung. (2019). Zwischenbericht ein Jahr KI-Strategie. https://www.bmwi.de/ Redaktion/DE/Downloads/XYZ/zwischenbericht-ein-jahr-ki-strategie.pdf?_blob=pub licationFile\&v=6. Zugegriffen: 18. Nov. 2020.

Domestika. (2020). https://www.domestika.org/en. Zugegriffen: 18. Nov. 2020.

EFI - Expertenkommission Forschung und Innovation. (2019). Gutachten zu Forschung, Innovation und technologischer Leistungsfähigkeit Deutschlands. Berlin: EFI.

Elements of AI. (2020). https://www.elementsofai.com/. Zugegriffen: 18. Nov. 2020.

Forum Bildung Digitalisierung. (2019). Reformstrategien Weltweit. Schule in der digitalen Welt. Berlin: Forum Bildung Digitalisierung e. V.

Goertz, L. (2014). Digitales Lernen adaptiv. Technische und didaktische Potenziale für die Weiterbildung der Zukunft. Gütersloh: Bertelsmann Stiftung.

H5P. (2020). https://h5p.org/. Zugegriffen: 18. Nov. 2020.

Hochschulforum Digitalisierung. (2020). Digital learning map. https://learnmap.hochschul forumdigitalisierung.de/. Zugegriffen: 18. Nov. 2020.

Hochschulrektorenkonferenz. (2020). HRK-Präsident zum Sommersemester. https://www. hrk.de/presse/pressemitteilungen/pressemitteilung/meldung/hrk-praesident-zum-som mersemester-4724/. Zugegriffen: 29. Apr. 2020. 
Hüther, O., Kosmützky, A., Asanov, I., Bünstorf, G., \& Krücken, G. (2020). Massive Open Online Courses after the Gold Rush: Internationale und nationale Entwicklungen und Zukunftsperspektiven. Hannover: Institutionelles Repositorium der Leibniz Universität.

Ifenthaler, D., \& Schumacher, C. (2016). Learning Analytics im Hochschulkontext. Wirtschaftswissenschaftliches Studium: WiSt, 45(4), 172-177.

KI-Campus - Die Lernplattform für Künstliche Intelligenz. (2020). https://ki-campus.org/ Zugegriffen: 18. Nov. 2020.

Kultusministerkonferenz. (2020). KMK: Sommersemester 2020 findet statt. https://www. kmk.org/aktuelles/artikelansicht/kmk-sommersemester-2020-findet-statt.html. Zugegriffen: 18. Nov. 2020.

LabXchange. (2020). Learning without limits. https://www.labxchange.org/. Zugegriffen: 18. Nov. 2020.

Mah, D., \& Büching, C. (2019). Künstliche Intelligenz in Studium und Lehre. Berlin: VDI/VDE Innovation + Technik GmbH.

Mah, D., Rampelt, F., Dufentester, C., Bernd, M., \& Gamst, C. (2020). Digitale Lernangebote zum Thema Künstliche Intelligenz. Überblicksstudie zu kostenlosen Online-Kursen auf deutschen Lernplattformen. Berlin: KI-Campus. https://ki-campus.org/publications/ studie-ki-online-kurse. Zugegriffen: 19. Okt. 2020.

mmb Institut GmbH. (2020). mmb-Trendmonitor 2019/2020. Essen: mmb Institut - Gesellschaft für Medien- und Kompetenzforschung mbH.

Rampelt, F., Schumacher, K., Zimmermann, V., Schmid, U., \& Staubitz, T. (2019). Von und mit der Maschine lernen. duz Wissenschaft \& Management (10), 36-39. https://www.res earchgate.net/publication/344404591. Zugegriffen: 19.Okt. 2020.

Schmid, U., Zimmermann, V., Baeßler, B., \& Freitag, K. (2018). Machbarkeitsstudie für eine (Inter-)Nationale Plattform für die Hochschullehre (Arbeitspapier, 33). Berlin: Hochschulforum Digitalisierung. https://doi.org/10.5281/zenodo.1408046.

Schulmeister, R. (2005). Lernplattformen für das virtuelle Lernen. Evaluation und Didaktik. Berlin: De Gruyter.

Seyda, S., Meinhard, D. B., \& Placke, B. (2018). Weiterbildung 4.0 - Digitalisierung als Treiber und Innovator betrieblicher Weiterbildung. IW-Trends. Vierteljahresschrift Zur Empirischen Wirtschaftsforschung, 45(1), 107-124.

Shah, D. (2019a). By the numbers: MOOCs in 2019 - Class central. https://www.classcent ral.com/report/mooc-stats-2019/. Zugegriffen: 18. Nov. 2020.

Shah, D. (2019b). Coursera's 2019: Year in review - Class central. https://www.classcentral. com/report/coursera-2019-year-review/. Zugegriffen: 18. Nov. 2020.

Squared Online. (2020). Der Online Marketing Kurs, der dich wirklich weiterbringt. https:// www.wearesquared.de/ Zugegriffen: 18. Nov. 2020.

Stifterverband für die Deutsche Wissenschaft e. V. (2020). Hochschulbarometer. https://www. hochschul-barometer.de/aktuell. Zugegriffen: 18. Nov. 2020.

University of Helsinki. (2019). The finnish 'Elements of AI' online course trains employees of the European union. https://www.helsinki.fi/en/news/data-science-news/the-finnishelements-of-ai-online-course-trains-employees-of-the-european-union. Zugegriffen: 18. Nov. 2020.

de Witt, C., Rampelt, F., \& Pinkwart, N. (Hrsg.) (2020). Künstliche Intelligenz in der Hochschulbildung. Whitepaper. Berlin: KI-Campus. https://doi.org/10.5281/zenodo.406 3722 . 
Zawacki-Richter, O., Marín, V. I., Bond, M., \& Gouverneur, F. (2019). Systematic review of research on artificial intelligence applications in higher education - Where are the educators? International Journal of Educational Technology in Higher Education, 16(1), 1-27.

Open Access Dieses Kapitel wird unter der Creative Commons Namensnennung 4.0 International Lizenz (http://creativecommons.org/licenses/by/4.0/deed.de) veröffentlicht, welche die Nutzung, Vervielfältigung, Bearbeitung, Verbreitung und Wiedergabe in jeglichem Medium und Format erlaubt, sofern Sie den/die ursprünglichen Autor(en) und die Quelle ordnungsgemäß nennen, einen Link zur Creative Commons Lizenz beifügen und angeben, ob Änderungen vorgenommen wurden.

Die in diesem Kapitel enthaltenen Bilder und sonstiges Drittmaterial unterliegen ebenfalls der genannten Creative Commons Lizenz, sofern sich aus der Abbildungslegende nichts anderes ergibt. Sofern das betreffende Material nicht unter der genannten Creative Commons Lizenz steht und die betreffende Handlung nicht nach gesetzlichen Vorschriften erlaubt ist, ist für die oben aufgeführten Weiterverwendungen des Materials die Einwilligung des jeweiligen Rechteinhabers einzuholen. 